\title{
Téoros
}

Revue de recherche en tourisme

\section{Le tourisme : un emploi d'avenir ?}

\section{Jean Stafford et Bruno Sarrasin}

Volume 20, numéro 1, printemps 2001

URI : https://id.erudit.org/iderudit/1071881ar

DOI : https://doi.org/10.7202/1071881ar

Aller au sommaire du numéro

\section{Éditeur(s)}

Université du Québec à Montréal

\section{ISSN}

0712-8657 (imprimé)

1923-2705 (numérique)

Découvrir la revue

Citer cette note

Stafford, J. \& Sarrasin, B. (2001). Le tourisme : un emploi d'avenir ? Téoros, 20(1), 72-74. https://doi.org/10.7202/1071881ar d'utilisation que vous pouvez consulter en ligne.

https://apropos.erudit.org/fr/usagers/politique-dutilisation/ 


\section{Conjo incture}

\section{Le tourisme : un emploi d'avenir ?}

\section{Jean Stafford et Bruno Sarrasin}

Cette chronique porte sur la conjoncture touristique. Elle propose un portrait simplifié d'une situation conjoncturelle dans le secteur du tourisme, à porrir des donnés disponibles.

e chômage est une notion autant politique qu'économique. Politique, puisque les gouvernements l'utilisent comme indicateur de retombées sociales de leurs décisions ; économique aussi, par l'arbitrage qu'il crée avec l'inflation, dans le but d'aboutir à une a croissance equilibre s (Silem, 1989). Sujet toujours d'actualité, l'évolution de l'emploi fait partie des indicateurs de santé de nos sociétés occidentales en révélant leurs forces et leurs faiblesses, selon les secteurs économiques. On parle souvent de pénurie de travailleurs dans les secteurs de pointe (technologie de l'information, aérospatiale, etc.) ; il est intếressant de souligner que, depuis quelques années, la forte demande de travailleurs se maintient dans l'industrie touristique.

Le Conseil canadien des ressources humaines en tourisme (CCRHT) est né d'une volonté de plusieurs associations - re= présentant un segment important de l'industrie touristique canadienne - de résoudre les problèmés liés au manque de travailleurs qualifiés dans ce domaine. Cette situation n'est pas propre au tourisme, mais elle est exacerbée par le caractère saisonnier de ce type de travail (Stafford et Sarrasin, 1999), ce qui rend très difficile la fidélité aux emplois de ce secteur. Depuis sa création, le CCRHT encourage la formation et la gestion des ressources humaines dans l'industrie touristique, de façon à réduire les conséquences des récessions et des reprises économiques sur la main-d'ceuvre. Chaque province possède maintenant son équivalent ${ }^{1}$.

La nécessité d'organiser une offre de formation cohérente et adaptée âl l'industrie touristique est d'autant nécessaire que la croissance économique des demières années, particulièrement depuis la seconde moitié des années 1990, ajoute de la pression sur les marchés du travail canadien et québécois. Dans un contexte de croissance, le taux de chômage est en baisse dans plusieurs secteurs, dont le tourisme. La conjoncture économique favorable chez nos voisins du Sud, de 1997 à 2000 , jumelée à une baisse marquée du dollar canadien par rapport au dollar américainin, a contribué à rếduire le déficit de la balance touristique au Canada et au Québec et à créer des emplois en nombre croissant.

Une caractéristique importante marque cependant le type d'emplois offert dans l'industrie touristique : un nombre important de ceux-ci se situe au premier échelon, c'est-à-dire que ce sont des emplois peu qualifiés et mal rémunérés. Ce type d'emplois procure une première expérience de travail à un Canadien sur trois et permet souvent aux étudiants de niveau post-secondaire d'obtenir un emploi d'été. Ces emplois constituent une grande partie de l'offre générée par l'industrie touristique, mème si l'on observe une demande de travailleurs de plus en plus qualifiés depuis dix ans.

En 1997, le CCRHT prévoyait la création de 306000 nouveaux emplois dans l'industrie d'ici 2005 . Il est important de noter que ces prévisions ont été faites en période de forte croissance, ce qui risque de changer au cours des prochaines années. Il est vrai qu'en période de croissance dans le secteur du tourisme, la demande de travailleur's qualifiés crée un mouvement ascendant des travailleurs, obligeant souvent les entreprises à assurer ellesmêmes la formation de leur main-d'auvre. Mais, comme le souligne le CCRHT,

Dans un tel contexte, les industries employant un grand nombre de travailleurs relativement moins competents sont davantage affectees, parce qu'il n'y a pas de bassin de travailleurs à un niveau moindre vers lequel elles pervent se wouner pour combler les nouveaux postes on ceux laissés vacants - le commerce au détal let le tourisme, par exemple (Commission canadienne du tourisme, $2001: 15$ ).

Le problème de pénurie demeure dans certains sous-secteurs de l'industrie touristique, dans la mesure où la capacité des entreprises à combler les nouveaux postes et à remplacer les travailleurs aux postes existants est de plus en plus menacée. Comme le propose le CCRHT, il existe plusieurs façons traditionnelles de réagir à une pénurie de main-d'ouvre et les mesures pour encourager le maintien en poste figurent généralement au premier rang. Ces mesures comprennent la rémunération, le perfection- 


\begin{tabular}{|c|c|c|c|c|c|c|}
\hline \multicolumn{7}{|c|}{ 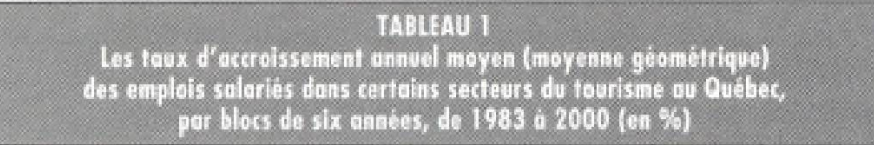 } \\
\hline Années & $\begin{array}{l}\text { Hobiels-motels } \\
\text { ef tamps } \\
\text { pour touristes }\end{array}$ & $\begin{array}{l}\text { Services } \\
\text { de la } \\
\text { restauration }\end{array}$ & $\begin{array}{l}\text { Tavernes, } \\
\text { bars et } \\
\text { boilies de } \\
\text { nwin }\end{array}$ & $\begin{array}{l}\text { Services } \\
\text { de voyages }\end{array}$ & $\begin{array}{l}\text { Trausport } \\
\text { aérien" }\end{array}$ & $\begin{array}{l}\text { Total des } \\
\text { salaries } \\
\text { de loes les } \\
\text { secteurs }\end{array}$ \\
\hline $1983-1988$ & 8,7 & 6,2 & 6,2 & 3,9 & 4,2 & 6,4 \\
\hline $1989-1994$ & $-6,6$ & $-1,8$ & -79 & 4,3 & $-5,3$ & $.3,3$ \\
\hline $1995-2000$ & 12,3 & 1,2 & 4,3 & 6,5 & 6,4 & 2,5 \\
\hline $1983-2000$ & 4,2 & 2,0 & 1.8 & 6,6 & 2,3 & 26 \\
\hline
\end{tabular}

- Services connexes au transport atrien compris.

Source : Sratietique Conodo

nement professionnel et la reconnaissance professionnelle, mais elles demeurent cotteuses pour les entreprises. Nous croyons qu'une pénurie de main-d'cuvre se maintiendra dans certains sous-secteurs du tourisme aussi longtemps que les conditions de travail s'appuieront sur un statut précaire et un niveau de rémunération sous la moyenne de l'industrie des services. L'évolution des emplois salaries dans certains secteurs du tourisme au Québec, depuis le début des annees 1980, permet de mieux saisir les périodes de croissance et les éventuelles situations de pếnurie.

\section{La situation des emplois salariés ${ }^{2}$ dans certains secteurs du tourisme au Québec}

Nous présentons au tableau I le taux d'accroissement annuel moyen des emplois salariés dans certains secteurs du tourisme au Québec, par blocs de six années, de 1983 à 2000 ; nos observations portent donc sur dix-huit années.

Nous pouvons remarquer, au tableau 1, une assez forte croissance, pour tous les secteurs étudiés, pendant la période 1983-1988. La période suivante, 1989-1994, a êté très difficile : la plupart des secteurs (à l'exception des * Services de voyages $*$ ) ont connu une décroissance. Dans la dernière période, 1995-2000, nous assistons à une reprise de la croissance des emplois salariés dans la plupart des secteurs ; pendant ces années, la décroissance des « Services de la restauration s s'est maintenue (avee une baisse annuelle de $-1,2$ \%). De 1983 a 2000 , les \& Services de voyages ont connu une croissance très soutenue $(6,6 \%$, sans période négative) ; ils sont suivis du secteur de l'hôtellerie (4,2\%), du * Transport aérien $*(2,3 \%)$, des $\propto$ Services de la restauration $\%(2 \%)$ et du secteur des $\approx$ Tavernes, bars et boîtes de nuit $\%$ $(1,8 \%)$.

\section{L'évolution indicielle des emplois salariés dans certains secteurs du tourisme}

Le graphique 1 illustre l'évolution indicielle des emplois salariés dans certains secteurs ${ }^{3}$ du tourisme au Québec, de 1992 à 2000 (la base 100 est 1990). Le secteur de l' hôtellerie est resté « collé * à la barre 100 de 1991 à 1997 ; en 1998,1999 et 2000 , l’indice est passé de 119 à 150 et à 181 respectivement. Les \& Services de voyages s ont progresse de façon continue de 1987 a 1997 (1'indice est grimpé de 111 à 165 ) ; par la suite il y a eu une certaine stagnation (les indices sont de 157 en 1998, de 160 en 1999 et de 156 en 2000). Pour le secteur du \& Transport aérien $s$, on constate une baisse de l'évolution indicielle de 1992 à 1997 ; l'année la plus basse est 1994 avec un indice de 77 ; de 1998 à 2000,1 'indice est passé de 108 à 122 , ce qui représente une importante progression dans un court laps de temps.

\section{Les mouvements cycliques des emplois salariés dans certains secteurs du tourisme}

Le graphique 2 montre les mouvements cycliques des emplois salariés dans certains secteurs du tourisme au Québec, de 1983 à 2000. Il est assez étonnant de voir que les mouvements cycliques du secteur de l'hôtellerie évoluent diamétralement à l'opposé des mouvements cyclique du secteur des a Services de voyages \%. Voyons ces mouvements cycliques pour chacun des secteurs :

- Dans le secteur de l'hôtellerie il y a un cycle négatif en 1983 et 1984, suivi d'un cycle positif qui s"étend de 1985 à 1990 ; par la suite, il y a une longue glissade négative de 1991 à 1998 ; finalement, en 1999 et en 2000 le cycle est à nouveau positif (alors que les indices sont respectivement à $118 \mathrm{et} \mathrm{à} \mathrm{141).}$

- Le secteur des a Services de voyages * connaît un cycle positif de 1983 à 1986, un cycle négatif en 1987-1988; un nouveau cycle positif de 1989 à 1991, puis cinq années de vaches maigres (de baisse) de 1993 à 1996. Par la suite, il y a une reprise de 1997 a 1999 et une nouvelle baisse en 2000 (coefficient cyclique à 96).

- Dans le secteur du a Transport aérien \%, on assiste à une baisse en 1983-1984, suivie de sept années de hausse, de 1985 à 1991 , puis de six années de dégringolade, de 1992 à 1997, et de trois années d'embellie, de 1998 à 2000.

Le cycle de I"hôtellerie est le plus important. puisque les indices oscillent de la valeur minimale de 73 à la valeur maximale de 141. Pour le secteur des a Services de voyages 5 la fourchette est plus serrée " les coefficients cycliques se situent de 68 à 124. Le secteur du * Transport aérien montre le cycle le plus faible : ses indices évoluent de 78 à 116 .

\section{Les mouvements saisonniers des emplois salariés dans certains secteurs du tourisme}

Le graphique 3 illustre les coefficients saisonniers des emplois salariés dans certains secteurs du tourisme au Québec, de 1996 à 2000. Les mouvements saisonniers sont assez puissants dans le domaine de l'hôtellerie : le coefficient le plus élevé est de 113 et le plus faible de $92^{4}$; les mois les plus élevés sont de mai à octobre et les plus faibles de janvier à mars et de novembre à décembre. Dans le secteur des a Services de voyages s et du * Transport aérien $\%$, les coefficients sont faibles et oscillent de 
GRAPHIOUE I

Ecrolution indiciallo des canplois sulatios

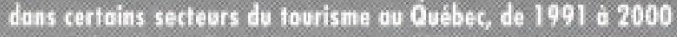

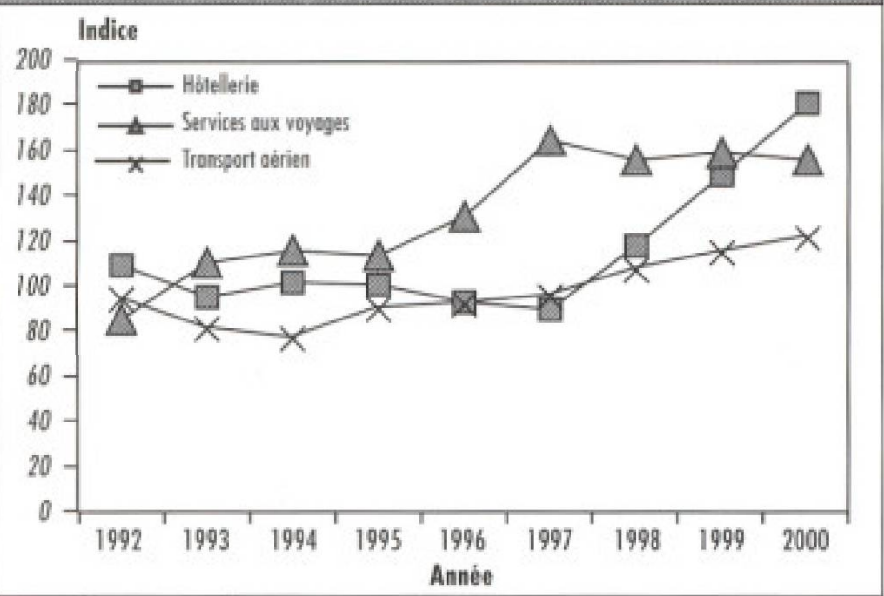

Soutcos: Statisique Canada

\section{GRAPHLUE 2}

Les mourenents cydiques des emplois salaries dens

certolns secteurs do tourisme cu Oesbec, de 1983 is 2000

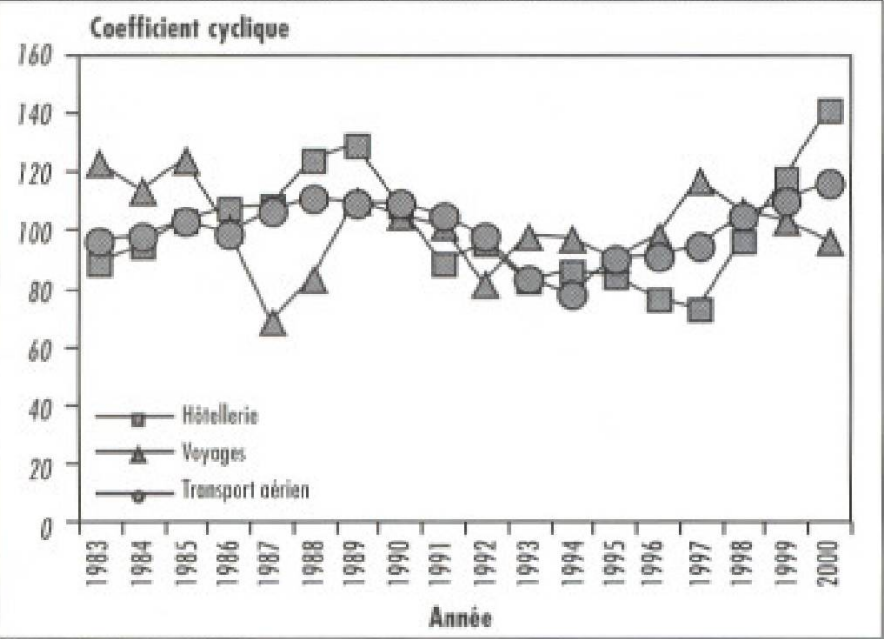

Souice : Statistique Canada

\section{GRAPHIOUI 3}

Les coeflicients suisonniers des emplois solerits

dans certains sedteurs do tourisme au Quther, de 1996 a 2000

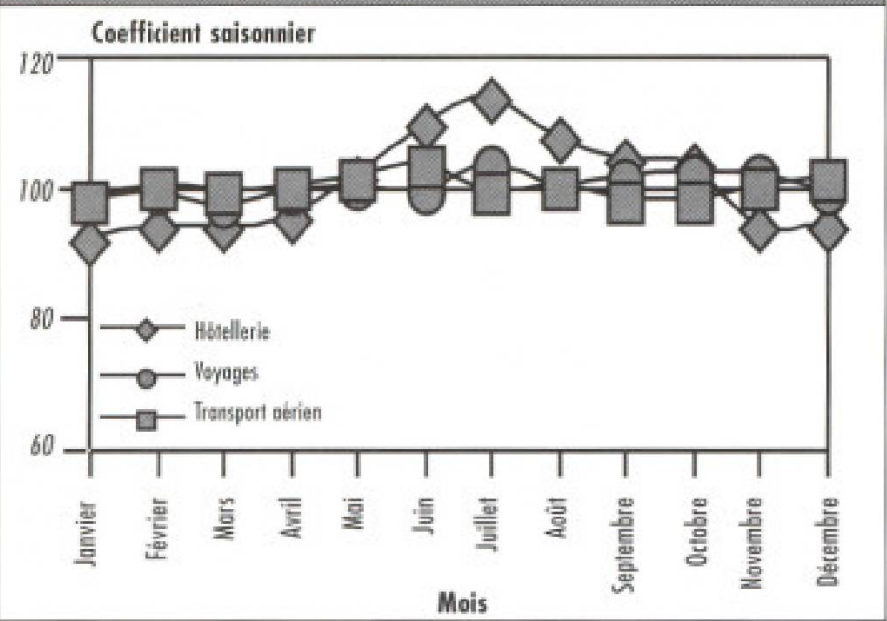

97 à 103 ; de janvier à juin, les coefficients de ces deux secteurs évoluent dans le même sens, mais de juillet à décembre, ils Evoluent en sens contraire (avec une faible amplitude).

\section{Du présent au futur}

A long terme - ici une période de dix-huit années -, nous remarquons que, dans les périodes cycliques négatives, les mouvements habituels des séries chronologiques en tourisme (les mouvements saisonniers et les mouvements irréguliers) ont tendance à s'exacerber et à prendre une forme cumulative. Les différents secteurs du tourisme sortent ă peine d'un long cycle négatif (de 1991 à 1997 environ) ; la déprime économique qui nous vient de nos voisins du Sud ${ }^{5} n^{\prime}$ est pas de bon augure. Selon une analogie médicale, quand les États-Unis ont le rhume, le Canada éternue... le Québec a une bonne grippe et l'industrie du tourisme souffre d'une dure bronchite.

Jean Stafford est professeur au Département d'études urbaines et touristiques de l'UQAM.

Bruno Sarrasin est chargé de cours au Département d'études urbaines et touristiques de l'UQAM.

\section{Notes}

1 Pour le Québec, voir www.cqrht.qc.ca

2 La principale source de nos donnes est tirée de la Matrice 4355 de CANSIM-Statistique Canada ; cette matrice s'intitule : Estimations des salariés par industrie, selon la C.T.I. de 1980, entreprises de toutes les tailles, données non-désaisonnalisés, Québec $\%$; les données annuelles sont décomposables par mois.

3 Nous avons conservé seulement des secteurs * purement * touristiques.

4 Ces bornes maximales et minimales (de l'offre) sont sans aucune mesure avec celles de la demande touristique.

5 Selon certains économistes, les États-Unis seraient déjà ơ techniquement $\%$ en récession.

\section{Bibliographie}

Commission canadienne du tourisme (2001), * La main-d"ouvre en tourisme \$, Communique, 5, (1).

Silem, Ahmed (1989), Introduction à l'analyse économique, Paris, Armand Colin.

Stafford, Jean, et Sarrasin, Bruno(1999), \& La saisonnalité dans les hötels du Québec : problèmes ou opportunités s, Téoros, 18 (1). 